\title{
Forward and backward recall: Different visuospatial processes when you know what's coming
}

\author{
Dominic Guitard ${ }^{1} \cdot$ Jean Saint-Aubin ${ }^{1} \cdot$ Marie Poirier $^{2} \cdot$ Leonie M. Miller $^{3}$ - Anne Tolan ${ }^{4}$
}

Published online: 25 July 2019

(C) The Psychonomic Society, Inc. 2019

\begin{abstract}
In an immediate memory task, when participants are asked to recall list items in reverse order, benchmark memory phenomena found with more typical forward recall are not consistently reproduced. These inconsistencies have been attributed to the greater involvement of visuospatial representations in backward than in forward recall at the point of retrieval. In the present study, we tested this hypothesis with a dual-task paradigm in which manual-spatial tapping and dynamic visual noise were used as the interfering tasks. The interference task was performed during list presentation or at recall. In the first four experiments, recall direction was only communicated at the point of recall. In Experiments 1 and 2, fewer words were recalled with manual tapping than in the control condition. However, the detrimental effect of manual tapping did not vary as a function of recall direction or processing stage. In Experiment 3, dynamic visual noise did not influence recall performance. In Experiment 4, articulatory suppression was performed on all trials and manual tapping was added on half of them. As in the first two experiments, manual tapping disputed forward and backward recall to the same extent. In Experiment 5, recall direction was known before list presentation. As predicted by the visuospatial hypothesis, when manual tapping was performed during recall, its detrimental effect was limited to backward recall. Overall, results can be explained by calling upon a modified version of the visuospatial hypothesis.
\end{abstract}

Keywords Short-term memory $\cdot$ Backward recall $\cdot$ Visuospatial hypothesis

\section{Introduction}

In a typical immediate serial recall task, participants recall lists of items immediately after their presentation, beginning with the first presented item and ending with the last one. In addition to this standard immediate serial recall task, there is a version in which participants recall items by beginning with the last presented item and ending with the first. This is known as backward recall. Although backward recall has been studied for over a century (see Blankenship, 1938, for an early literature review and Donolato, Giofré, \& Mammarella,

Jean Saint-Aubin

jean.saint-aubin@umoncton.ca

1 École de Psychologie, Université de Moncton, Moncton, New Brunswick E1A 3E9, Canada

2 University of London, London, UK

3 University of Wollongong, Wollongong, NSW, Australia

4 University of Southern Queensland, Toowoomba, QLD, Australia
2017, for a recent literature review), it is less well understood than forward recall (Lewandowsky \& Farrell, 2008). Nevertheless, backward recall performance and error types have helped to test the predictions and limits of models in the area and hence contribute to theoretical developments (Beaudry, Saint-Aubin, Guérard, Pâquet, 2018; Donolato et al., 2017; Henson, 1998; Oberauer et al., 2018; Page \& Norris, 1998; Hulme, Roodenrys, Schweickert, Brown, Martin, \& Stuart, 1997; Saint-Aubin, Guérard, Chamberland, \& Malenfant, 2014). Furthermore, backward recall is one of the most frequently used methods in clinical and neuropsychological research and assessment. In effect, backward recall is incorporated in widely used tests. For instance, backward digit span is used in Wechsler Intelligence Scales (Wechsler, 2008), the Mini Mental Status Examination (Folstein, Folstein, \& McHugh, 1975), the British Abilities Scales II (Elliot, 1996), and the Working Memory Test Battery for Children (Pickering \& Gathercole, 2001). In addition, compared to forward recall, backward recall better predicts mild cognitive impairment (Muangpaisan, Intalapaporn, \& Assantachai, 2010), agerelated cognitive decline (Bopp \& Verhaeghen, 2005), and academic learning (see, e.g., Bull, Espy, \& Wiebe, 2008; 
Gathercole, Pickering, Knight, \& Stegmann, 2004). Because backward recall is a valuable tool for a variety of domains, better understanding processes it calls upon would have a broad impact.

The latter aim is challenging because benchmark memory phenomena - well-established in forward recall - are not systematically reproduced with backward recall (see, e.g., Baker, Tehan, \& Tehan, 2012; Beaudry, et al., 2018; Bireta et al., 2010; Guérard, Saint-Aubin, Burns, \& Chamberland, 2012; Hulme et al., 1997; Li \& Lewandowsky, 1993; Ritchie, Tolan, Tehan, Goh, Guérard, \& Saint-Aubin, 2015; Rosen \& Engle, 1997; Surprenant, Brown, Jalbert, Neath, Bireta, \& Tehan, 2011; Tehan \& Mills, 2007; Walker \& Hulme, 1999). In order to account for these inconsistencies, a visuospatial hypothesis has been suggested (Hermelin \& O'Connor, 1973; Li \& Lewandowsky, 1993, 1995; O’Connor \& Hermelin, 1973; St Clair-Thompson \& Allen, 2013). The current study was designed to provide a comprehensive investigation of said visuospatial interpretation.

The visuospatial hypothesis can be described as a dualprocess view of immediate serial recall, where forward and backward recall are thought to call upon qualitatively distinct retrieval processes (Li \& Lewandowsky, 1993, 1995; Reynolds, 1997; St Clair-Thompson \& Allen, 2013). For instance, using factor analyses, Reynolds (1997) found that the forward and backward digit spans of Weschler's intelligence tests loaded on distinct factors (but see Colom, Abad, Rebello \& Shih, 2005). Reynolds concluded that contrary to forward recall, "backward recall may also invoke, for many individuals, visuospatial imaging processes even for ostensibly verbal materials such as letters" (p.39). In line with those individual differences results, Hoshi et al. (2000) reported neuroimaging evidence of the implication of visuospatial codes in backward digit span. Using an optical tomographic imaging system, they found stronger activation in the dorsolateral prefrontal cortex, associated with visuospatial imagery, in backward than in forward digit span. Hoshi et al. concluded that visuospatial imagery is an efficient strategy for backward digit span but not for forward span (see also Gerton et al., 2004). A similar conclusion was reached in previous studies with brain-damaged populations (e.g., Hanley, Young, \& Pearson, 1991; Vallar \& Papagno, 1986). For instance, Rudel and Denckla (1974) found that forward digit span was significantly lower for patients with damage to the left hemisphere compared to a control group, while backward digit span was significantly lower for patients with brain injury to the right hemisphere, associated with the visuospatial code, than for the control group.

Using an experimental approach in which recall direction was only indicated at recall, Li and Lewandowsky (1995) reported that backward, but not forward, recall was impaired by presenting the to-be-remembered items at various spatial locations on the screen. Although fitting well with the visuospatial hypothesis, this finding is surprising in light of results about binding of verbal and visuospatial information. For instance, Guérard, Tremblay, and Saint-Aubin (2009) sequentially presented phonologically similar and dissimilar letters at different locations on a computer monitor. Participants either recalled the letters in their order of presentation or the spatial locations at which the letters had appeared. Results showed a detrimental effect of phonological similarity on recall of locations suggesting binding of visuospatial and verbal information in forward recall, even when it is detrimental to performance.

In another experiment, Li and Lewandowsky (1995) manipulated visual similarity by contrasting lists of visually similar letters (e.g., $\mathrm{O}$ and $\mathrm{Q}$ ) with lists of visually distinct letters (e.g., $\mathrm{X}$ and B). Results revealed a beneficial effect of visual similarity in backward, but not in forward, recall; the latter results with visual similarity are intriguing. In effect, robust and reliable detrimental effects of visual similarity have subsequently been reported in forward recall of words (e.g., fly, cry, dry vs. guy, sigh, lie; Logie, Della Sala, Wynn, \& Baddeley, 2000; Logie, Saito, Morita, Varma, \& Norris, 2016; Saito, Logie, Morita, \& Law, 2008) and of easily nameable line drawings or abstract matrix patterns (Poirier, Saint-Aubin, Musselwhite, Mohanadas, \& Mahammed, 2007).

St Clair-Thompson and Allen (2013) also tested the visuospatial hypothesis with a dual task procedure allowing them to selectively impair encoding or retrieval processes. More specifically, participants heard sequences of digits for an immediate forward or backward digit recall task and had to respond verbally. In all their experiments, recall direction and interference conditions were blocked and pre-cued. In addition, they introduced, either at encoding or at retrieval, the n-back task or Dynamic Visual Noise (DVN), an array of squares that randomly switch between black and white and are known to interfere with visuospatial processing (Chubala et al., 2018). In the n-back task, black circles randomly appeared one at a time on the screen. Each circle could appear at one of nine possible locations. Participants were asked to indicate the location currently occupied by the circle (0-back) or the position occupied by the circle two trials before (2-back). During the digit memory task, the n-back task was performed at encoding or at recall. At encoding, the n-back task had the same detrimental effect for both recall directions. However, at retrieval, the nback task had a greater impact on backward than on forward recall. This pattern of results was interpreted in favor of the visuospatial hypothesis. However, the n-back task is a complex task calling upon multiple processes, including executive processes (see, e.g., Chatham, Herd, Brant, Hazy, Miyake, O'Reilly, \& Friedman, 2011). In other words, even if the nback task required memory for spatial locations, it is impossible to know if its detrimental effect was due to the spatial, the executive control, or any other components of the task.

It could be argued that the experiments in which DVN was introduced overcame the limitations observed with the 
n-back task. In support of this view, results with DVN were similar to those with the n-back task: DVN hindered backward, but not forward, performance when it was introduced at recall. Although fitting well with the visuospatial hypothesis, these results must be interpreted with caution. In effect, in two experiments, the list length was not the same in the forward and backward recall conditions. This situation is not optimum because previous studies have shown that the magnitude of some memory effects, such as the phonological similarity effect, was modulated by list length (e.g., Salamé \& Baddeley, 1986). Furthermore, when list length was equated, performance was at ceiling in the forward recall condition. Therefore, it is impossible to know if the observed interaction reflects a real selective influence of DVN on backward recall or simply a ceiling effect in forward recall.

In the present experiments, we tested the role of visuospatial representations in backward recall by using a dualtask procedure. Manual spatial tapping was used to interfere with spatial representations because its detrimental effect on spatial representations is well established. In effect, manual spatial tapping has been found to interfere with visuospatial and verbal forward recall, although the effect was larger with visuospatial materials (Guérard \& Tremblay, 2008; Guitard \& Saint-Aubin, 2015; Jones, Farrand, Stuart, \& Morris, 1995; Meiser \& Klauer, 1999; Poirier, Yearsley, Saint-Aubin, Fortin, Gallant, \& Guitard, 2019). In Experiment 1, participants memorized lists of seven words for an immediate serial recall task. Recall direction varied randomly from trial to trial and was only indicated at retrieval. The direction of recall was specified just before retrieval to reduce the possibility that encoding would be different in each recall condition. Tapping was required at encoding or recall. In Experiment 2, words were replaced by digits, known for their intrinsic visuospatial representation (see Spatial-Numerical Association of Response Codes (SNARC) effect; Dehaene, Bossini, \& Giraux, 1993). In Experiment 3, DVN was used as the distractor and in Experiment 4 articulatory suppression was added to tapping. In Experiment 5, recall direction was blocked and known in advance to mimic the conditions used in psychometric tests. The inclusion of this condition allows testing whether specific encoding processes would be called upon when expecting backward recall and whether they would modulate retrieval processes.

\section{Experiment 1}

In Experiment 1, we tested the visuospatial hypothesis by combining immediate serial recall of words and manual spatial tapping as a secondary task. Manual spatial tapping was required at encoding or at recall. According to previous findings and to the visuospatial hypothesis, a larger detrimental effect of tapping should be observed in backward than in forward recall, when it is performed during the recall stage ( $\mathrm{St}$ Clair-Thompson \& Allen, 2013).

\section{Method}

Participants Forty undergraduate students (33 women and seven men) from Université de Moncton volunteered to participate for course credits. All participants reported normal or corrected-to-normal vision and were French speakers.

Materials The stimuli were monosyllabic French words with a mean frequency count of 141.66 occurrences per million ( $\mathrm{min}$. $=0$, max. $=8,795)$ according to Lexique (New, Pallier, Brysbaert, \& Farrand, 2004). The 392 words were randomly assigned to 56 lists, each with seven words, with the constraints that a word could only be used in one list and no words within a list could rhyme. All words were presented in black, lowercase, 28-point Arial font, at the center of the computer screen. For the spatial-manual interference task, using their non-dominant hand, participants were required to press the 11 outside keys of the numeric keypad one at a time counter-clockwise at a rate of two taps per second (see, Jones et al., 1995). The experiment was controlled with EPrime 2.0 with a resolution of $800 \times 600$ pixels and the stimuli were presented on a $47.72-\mathrm{cm}(18$-in.) screen.

Design A $2 \times 2 \times 2 \times 7$ mixed-design was implemented with the locus of the interference (presentation, recall) as the between-participants factor and recall direction (forward, backward), interference (none, manual spatial tapping) and serial position (1-7), as the repeated-measures factors. Conditions and lists were randomized for each participant. In addition, words within each list were presented in a different random order for each participant.

Each participant underwent one block of 56 experimental trials preceded by four practice trials. There were 14 experimental trials and one practice trial for each of the four possible conditions (forward control, forward manual spatial tapping, backward control, backward manual spatial tapping). As mentioned above, the order of the four practice trials and the 56 experimental trials were randomized for each participant.

Procedure All participants were tested individually in one experimental session lasting approximately $30 \mathrm{~min}$. The participant sat approximately $60 \mathrm{~cm}$ from the screen; the experimenter was present throughout the session to ensure compliance with the instructions. The numeric keypad was placed in the center of the table space just in front of the participants. Participants in the interference at encoding condition first saw the instructions for the interference task during 1,500 ms. The word "frappe" (tapping) in blue 
signalled the requirement of pressing the keys of the numeric keyboard during the presentation, and the word "fixe" (stay still) in blue required participants to stay still during the presentation of the words. Immediately after, the seven to-be-remembered words were presented at a rate of one word per second (1,000 ms on, $0 \mathrm{~ms}$ off) on the center of the screen. After the presentation of the last word, all words reappeared simultaneously in random order on three lines on the center of the screen and stayed on the screen for the recall phase. Recall direction was indicated in the upper part of the screen in capital letters by the word "NORMAL" in blue (normal) for the forward recall condition or by the word "INVERSÉ" in red (backward) for the backward recall condition just above the symbol $=>$ or $<=$ presented in black. For participants in the interference at recall condition, the procedure was exactly as described above, except that instructions for the interference task were displayed after the presentation of the last to-beremembered word. Participants began tapping as soon as the instructions were displayed and continued until recall was completed. In both interference conditions, in the forward recall condition, participants were expected to recall the list of words from the first to the last word that had been presented. In the backward recall condition, participants were expected to recall the list from the last to the first presented word. Participants were instructed to recall the items by saying them aloud and by saying "passe" (skip) whenever they did not know the word at a given serial position. Participants were not allowed to backtrack in order to modify a previous response. Responses were recorded with a digital recorder for later codification. Participants initiated the next trial by pressing the left button of the mouse.

\section{Results}

A strict serial recall criterion was used. With this criterion, words must be recalled in their presentation position to be considered correct. For all analyses, the proportion of correct recall was assessed as a function of serial position, recall direction (forward vs. backward), interference (none vs. tapping), and the locus of the interference (presentation vs. recall). All analyses of variance (ANOVAs) are reported in Appendix Table 1. In all experiments, the .05 level of significance was adopted, and the Greenhouse-Geisser correction was applied when the sphericity criterion was violated.

As shown in Figs. 1 and 2, participants were significantly better at recalling words in the control than in the manual tapping condition. Most importantly, tapping produced a similar modest memory decrement in both forward and backward recall conditions whether it occurred at encoding or at recall. The effect of tapping was slightly larger when performed at encoding than at recall. Reflecting those trends, the ANOVA reported in Appendix Table 1 revealed that recall accuracy was significantly lower in the tapping $(M=.40, S D=.28)$ than in the control condition $(M=.43, S D=.29)$. As usual, the main effect of serial position was significant as well as its interaction with recall direction. In addition, there was a significant interaction between serial position, recall direction, and the locus of interference. This interaction is not of particular theoretical interest and will not be further discussed. None of the other interactions reached significance.

The non-significant interaction between recall direction and interference, and the non-significant interaction between recall direction, interference, and its locus are of particular interest, $F=1.27, p=.27 ; F<1$, respectively. Given the theoretical importance of the three-way interaction between recall direction, interference, and its locus, we examined the size of the tapping effect for both recall directions (forward vs. backward) and interference groups (presentation vs. recall) by subtracting the proportion of correct recall in the interference condition from the proportion of correct recall in the control condition. Contrary to the predictions derived from the visuospatial hypothesis, when the interference occurred at encoding, the effect of tapping was slightly larger in the forward $(M=.06, S D=$ $.09)$ than in the backward recall $(M=.03, S D=.07)$ condition. When interference occurred at recall, the effect of tapping was similar in both recall directions (forward: $M=$ $.02, S D=.08$; backward $=M=.02, S D=.07$ ).

Bayesian paired-samples $t$-tests Because it is not possible to argue for a null effect with null hypothesis testing (Jeffreys, 1961), and because the null effect of recall direction on the size of the tapping effect is critical for evaluating the visuospatial hypothesis, we computed Bayesian paired-samples $t$ tests using JASP (Jasp Team, version 0.9.1.0). For all Bayesian paired-samples $t$-tests, the results were interpreted with the Bayes factor (BF) approach (Jeffreys, 1961; Kass \& Raftery, 1995), which provides evidence in support of the null hypothesis (subscripted as 01 ) or the alternative hypothesis (subscripted as 10). The BF interpretation is based on the benchmarks taken from Kass and Raftery (1995): BF $<3$ indicates weak or anecdotal evidence; $3 \leq \mathrm{BF}<20$ indicates positive evidence; $20 \leq \mathrm{BF}<150$ indicates strong evidence; and $\mathrm{BF}>150$ indicates very strong evidence. For all Bayesian paired-samples $t$-tests, we set the Cauchy prior width to JASP default value $r=0.707$ (see Wagenmakers et al., 2018, 2018, for examples, rationale, and a comprehensive review of Bayesian analysis). Given that the visuospatial hypothesis is directional (backward detrimental effect $>$ forward detrimental effect), we incorporated the order restriction (backward $>$ forward) for all analyses unless otherwise mentioned. For simplicity, we interpreted the Bayes factor in function of the null model (the backward detrimental effect is not greater than the forward detrimental effect). 

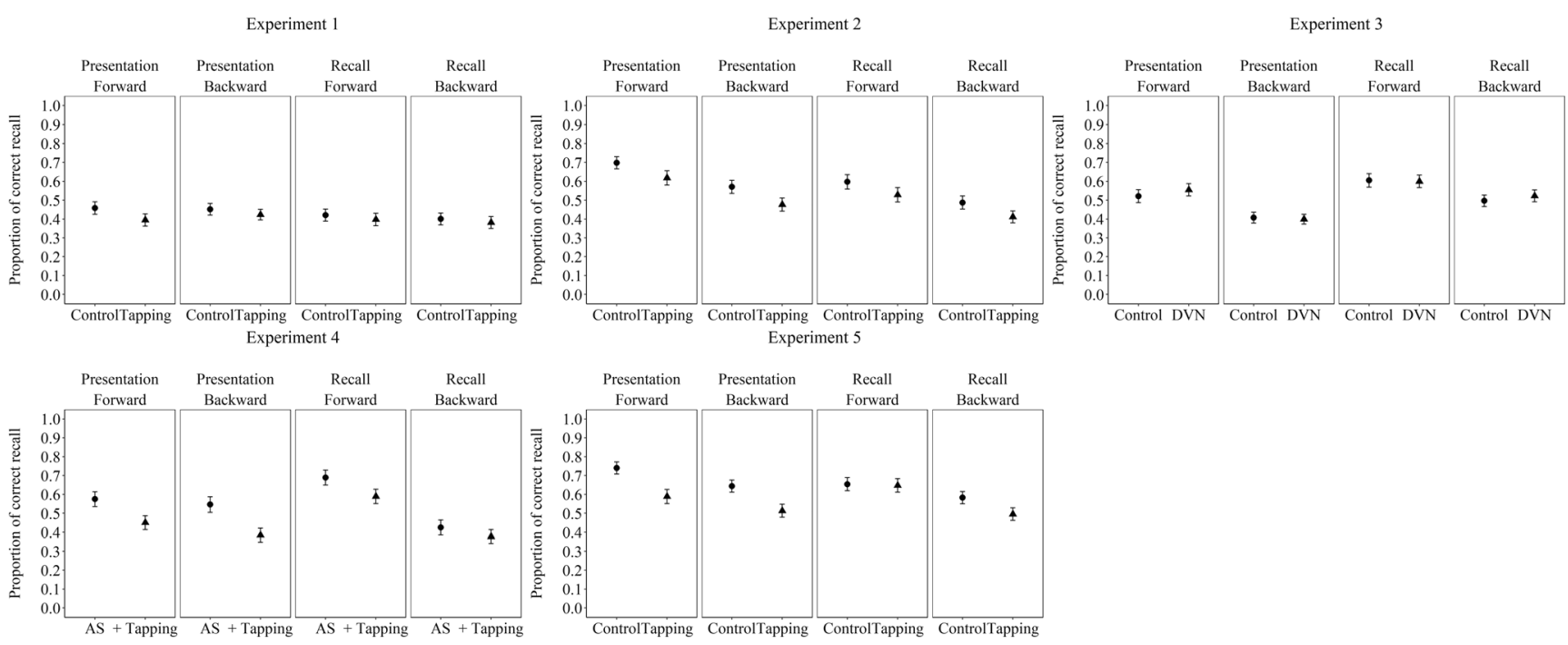

Fig. 1 Proportion of correct recall for Experiments 1, 2, 3, 4, and 5 as a function of recall direction (forward vs. backward), locus of the interference (presentation vs. recall), and interference task (none vs.

spatial manual tapping or Dynamic Visual Noise). Error bars represent 95\% within-participant confidence intervals computed according to Morey's (2008) procedure

Separate Bayesian $t$-tests were conducted for each group under the hypothesis that tapping effect should be larger in the backward than in the forward recall condition. Evidence in favor of the null hypothesis (backward tapping effect is not greater than the forward tapping effect) was positive when tapping was performed at presentation, $\mathrm{BF}_{01}=9.42$, or at recall $\mathrm{BF}_{01}=4.84$.

\section{Discussion}

Overall, recall accuracy was lower in the presence of manual tapping than in the control condition, and this pattern was observed when tapping was performed at encoding or at recall. The effect of tapping replicates previous findings observed when tapping was required during presentation and a

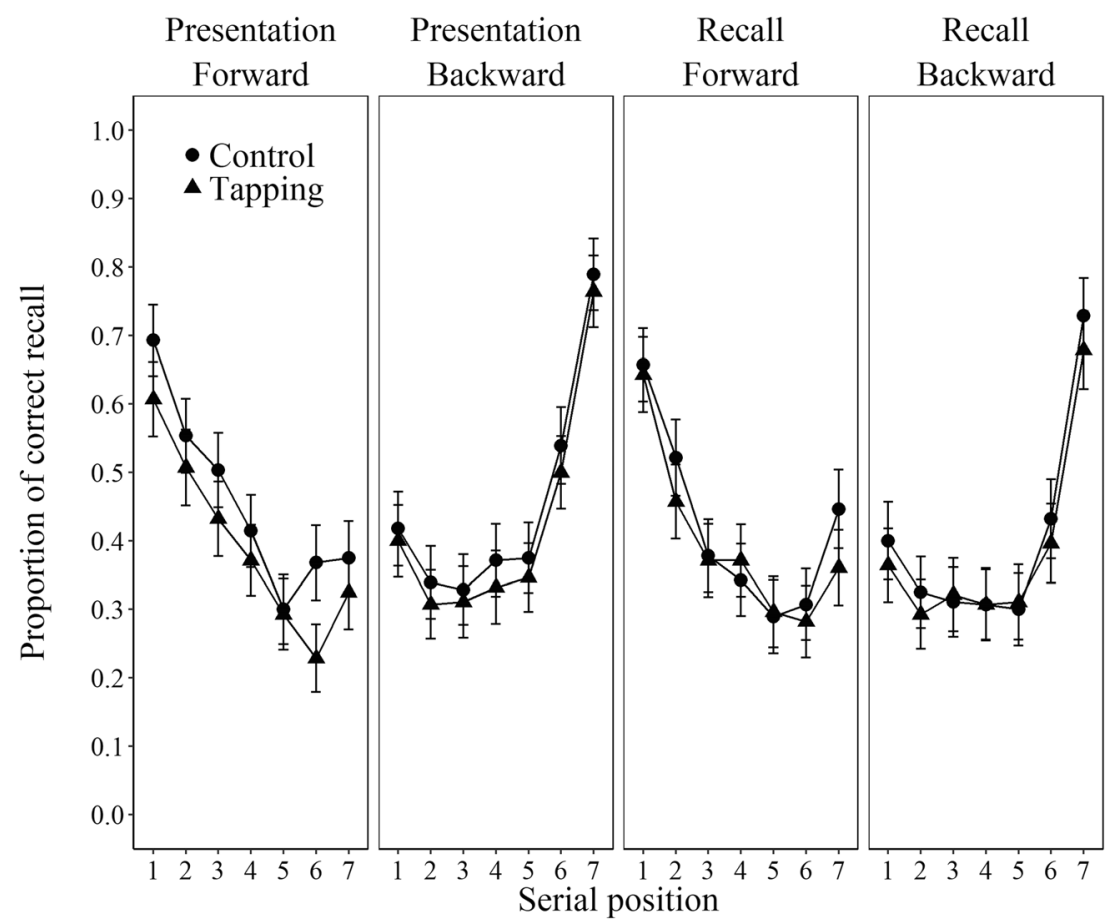

Fig. 2 Proportion of correct recall for Experiment 1 as a function of recall direction (forward vs. backward), locus of the interference (presentation vs. recall), interference task (none vs. spatial manual tapping), and serial position (1-7). Error bars represent 95\% within-participant confidence intervals computed according to Morey's (2008) procedure 
10-s retention interval (Guitard \& Saint-Aubin, 2015; Jones et al., 1995), or during presentation only (Guérard \& Tremblay, 2008). Furthermore, at the descriptive level, the detrimental effect of tapping during encoding was superior to recall, which extends previous findings of larger tapping effects during encoding than retention (see, e.g., Meiser \& Klauer, 1999). The similar detrimental effect of tapping in forward and backward recall and particularly for the group performing tapping at recall is contrary to the pattern of results reported by St Clair-Thompson and Allen (2013). In Experiment 2, we examined one possible cause for this discrepancy.

\section{Experiment 2}

St Clair-Thompson and Allen (2013) used digits as the to-beremembered items, while in Experiment 1 we used words. Despite the fact that words and digits are both verbal items, it can be argued that they do not involve visuospatial representations to the same extent as digits. Compared to most words, digits benefit from intrinsic visuospatial representations as evidenced by the SNARC effect (see, e.g., Dehaene, Bossini, \& Giraux, 1993). In parity-judgment tasks, response latency differences associated with the right and left hand are negatively correlated with number magnitudes. Therefore, the greater involvement of visuospatial representations in backward recall may only be observed with digits. We tested this hypothesis by using the same design as in Experiment 1, but we replaced words with digits.

\section{Method}

Participants Forty undergraduate students (34 women and six men) from Université de Moncton volunteered to participate in exchange for course credits. All reported normal or corrected-to-normal vision and were French speakers. None had participated in the previous experiment.

Materials and procedure The materials and procedure were the same as those used in Experiment 1 except that lists were made by randomly selecting seven digits with the constraint that a digit could only appear once within a list.

\section{Results}

As shown in Figs. 1 and 3, results are virtually the same as those found in Experiment 1: Recall was lower in the spatial manual tapping than in the control condition, and the magnitude of this effect was similar in forward and backward recall. As shown in Appendix Table 1, the ANOVA revealed that recall performance was significantly lower in the interference $(M=.51 S D$ $=.32)$ than in the control condition $(M=.59 S D=.31)$ and participants were better at recalling the digits in the forward $(M$ $=.61 S D=.31)$ than in the backward recall condition $(M=.49$ $S D=.32$ ). However, there was no significant interaction between recall direction and interference or between recall direction, interference, and the locus of the interference, both $F<1$.

Bayesian paired-samples $t$-tests As in Experiment 1, under the assumption that the size of the detrimental effect of spatial manual tapping should be larger in backward than in forward recall, we computed Bayesian paired-samples $t$-tests between recall direction for each interference group (presentation or recall). As in Experiment 1, the size of the interference score was obtained by subtracting the proportion of correct recall in the interference condition from the proportion of correct recall in the control condition. Like in Experiment 1, evidence in favor of the null hypothesis (backward tapping effect is not greater than the forward tapping effect) was positive when tapping was performed at presentation, $\mathrm{BF}_{01}=3.08$, or at recall $\mathrm{BF}_{01}=3.71$.

\section{Discussion}

Results of Experiment 2 revealed the typical detrimental effect of tapping on performance. More importantly, as can be seen in the Appendix Table 1 by comparing the size of the tapping effect in Experiments 1 and 2, digits $\left(\eta_{p}^{2}=.59\right)$ were more disrupted by manual spatial tapping than words $\left(\eta_{p}^{2}=.28\right)$. This difference fits nicely with the hypothesis that digits benefit from better visuospatial representations than words, as previously shown with the SNARC effect (Dehaene et al., 1993). However, even if digits were more sensitive to spatial manual tapping, the latter was equally disruptive in backward than in forward recall. These results diverge from those of St Clair-Thompson and Allen (2013), who have shown a larger detrimental effect of DVN on backward than on forward recall. The nature of the interference task may account for discrepancies across studies.

\section{Experiment 3}

It has been suggested that spatial manual tapping interferes with motor actions such as oculomotor movements used to support the retention of visuospatial information (see Tremblay, Saint-Aubin, \& Jalbert, 2006). However, in the context of backward recall, by interfering with spatial representations, tapping may not be the best secondary task. Instead, the DVN procedure developed by Quinn and McConnell (1996) and used by St Clair Thompson and Allen (2013) may be more appropriate. Quinn and McConnell showed that DVN can interfere with the encoding, maintenance, and retrieval of purely visual information in working memory. They tested participants with the typical forward recall procedure. DVN interfered with performance when participants recalled the word 


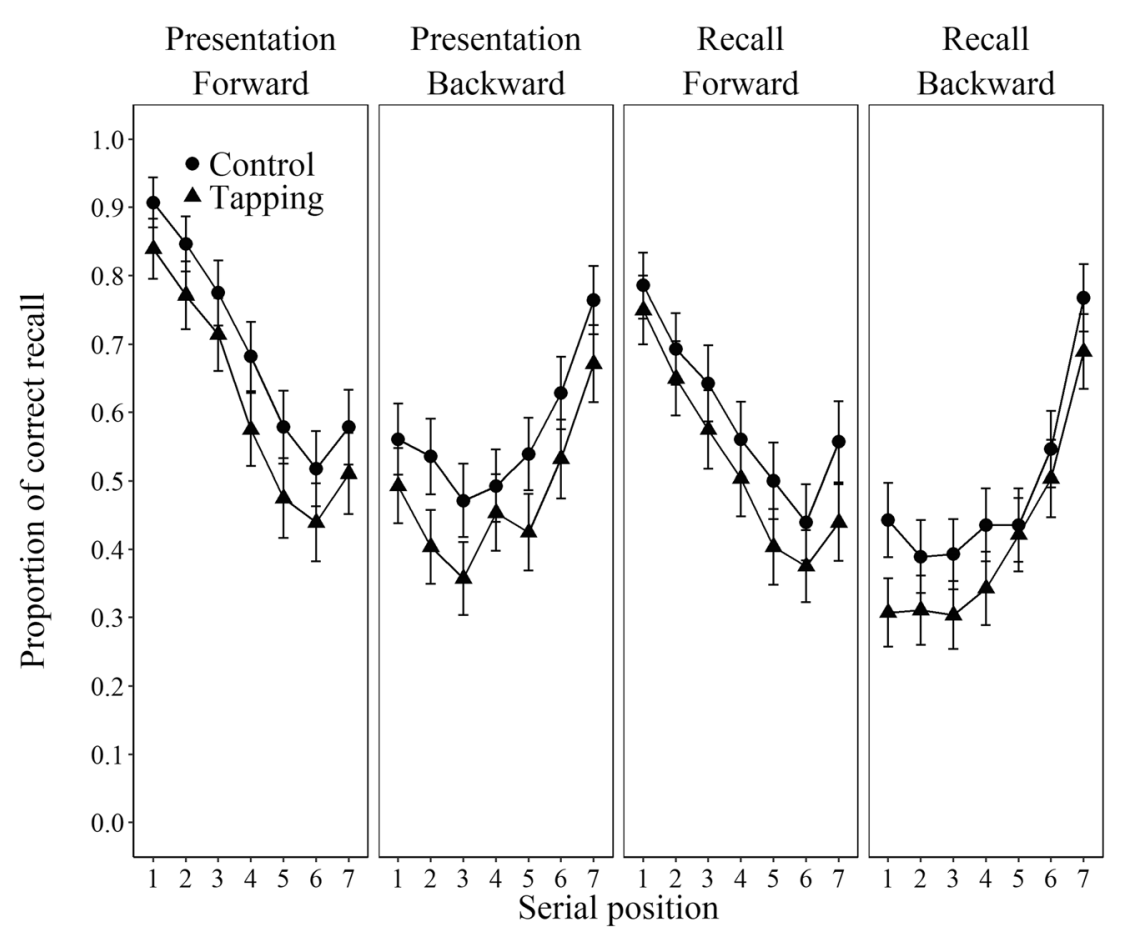

Fig. 3 Proportion of correct recall for Experiment 2 as a function of recall direction (forward vs. backward), locus of the interference (presentation vs. recall), interference task (none vs. spatial manual tapping), and serial

lists under elaborated visual mnemonic mediation instructions. More precisely, participants first learned a list of ten pegwords ("one is a bun, two is a shoe," etc.) and then generated an image for each item. They were then asked to create a visual image for each word from the list as it was presented and to integrate it with the image of the pegword. Recall was based on this integrated image. It should be noted that DVN had no effect when participants were not using this exhaustive visual strategy and were simply rehearsing the list. In line with those results, St Clair-Thompson and Allen used DVN in three experiments. As predicted, a selective detrimental effect was observed in backward recall when DVN occurred at recall. However, as mentioned above, in two experiments, list length varied as a function of recall direction, and in Experiment 5, when list length was equated, performance was at ceiling in forward recall impeding the possibility of observing a DVN, or any other effect. In Experiment 3, we introduced DVN as was used by St Clair-Thompson and Allen to further test the visuospatial hypothesis. The design was the same as in Experiments 1 and 2, but we replaced spatial manual tapping by DVN and used auditory presentation.

\section{Method}

Participants Forty undergraduate students (28 women and 12 men) from Université de Moncton volunteered to participate in exchange for course credits. All reported normal or position (1-7). Error bars represent 95\% within-participant confidence intervals computed according to Morey's (2008) procedure

corrected-to-normal vision and were French speakers. None had participated in the previous experiments.

Materials and procedure The materials and procedure were the same as those used in Experiment 2, except for the following changes. The sequence of digits was presented aurally through headphones instead of visually on the computer screen. The presentation of digit sequences began 1,000 ms after the instructions offset and digits were presented at a rate of one digit per second. Audio files were generated using Google translate. We used the French pronunciation of the digits. Audio recordings were created with SoundTap Streaming audio Recorder 2.31 and sound files were edited with WavePad Sound Editor 5.96. Digits were not represented at recall. Recall instructions were the same as those used in Experiment 2 except that a 10-s time limit was added. The interference task was changed for DVN. DVN consisted of a grid of $80 \times 80$ black and white cells (for a similar procedure, see Quinn \& McConnell, 1996). At any given time, 50\% of the cells were white and $50 \%$ were black. The DVN was presented over $8,000 \mathrm{~ms}$ and the sequence of grid changes was the same for all participants. Participants were instructed to look at the center of the computer screen throughout the experiment and the grid changed every $1,000 \mathrm{~ms}$.

Participants pressed the spacebar to initiate a trial. For the encoding interference group, DVN or a white screen began immediately after the participant pressed the spacebar and ended $8,000 \mathrm{~ms}$ later as recall instructions appeared. 
Participants were given $10 \mathrm{~s}$ to respond orally. At the end of the recall period, a message was displayed on the screen indicating that the next trial can be initiated by pressing the spacebar. For the recall interference group, the presentation of DVN or a white screen with three question marks began $1,000 \mathrm{~ms}$ after the presentation of the last to-remembereditem. Participants were instructed to begin recall only after seeing the presentation of the question marks or the beginning of the DVN. Participants were also instructed to look at the center of the computer screen throughout the experiment.

\section{Results}

As can be seen in Figs. 1 and 4 and as confirmed by the ANOVA reported in Appendix Table 1, like Experiment 2, participants were better at recalling the digits in the forward $(M=.57 S D=.31)$ than the backward recall condition $(M=.46$ $S D=.28$ ), and the main effect of serial position was significant. However, DVN did not hinder recall performance and did not interact with recall direction or with the locus of interference.

Bayesian paired-samples $t$-tests As in the previous experiments, for each locus of the interference task (encoding or recall), we computed Bayesian paired-sample $t$-tests on the size of the interference task effect with the assumption that the effect of the interference task (DVN in this experiment) would be larger in backward than forward recall condition. Results revealed anecdotal evidence in favor of the null hypothesis, $\mathrm{BF}_{01}=0.24$, when the interference occurred during the presentation, and positive evidence, $\mathrm{BF}_{01} 7.39$, when the interference occurred at recall. It should be noted that the lack of evidence when DVN was introduced at encoding is due to a reverse DVN effect with worse recall performance of control lists than of DVN lists (for a similar trend, see Experiment 3 of Chubala et al., 2018).

\section{Discussion}

Current results are in line with those of Quinn and McConnell (1996), who developed the DVN procedure. They only found a detrimental effect of DVN when participants were forced to use an elaborated visual strategy. Similar results were observed by St Clair-Thompson and Allen (2013), who only found a DVN effect among the subset of participants using a visual strategy while performing backward recall with DVN presented at recall. Before concluding that spatial interference does not hinder backward more than forward recall, a further test is needed to rule out a strategy-based hypothesis.

\section{Experiment 4}

In immediate memory tasks, participants may use alternative strategies to meet task demands (see, e.g., Logie, 2018; Miles, Jones, \& Madden, 1991; Watkins, LeCompte, \& Kim, 2000). Perhaps variations in strategy could account for the findings reported herein. According to this view, even if backward recall

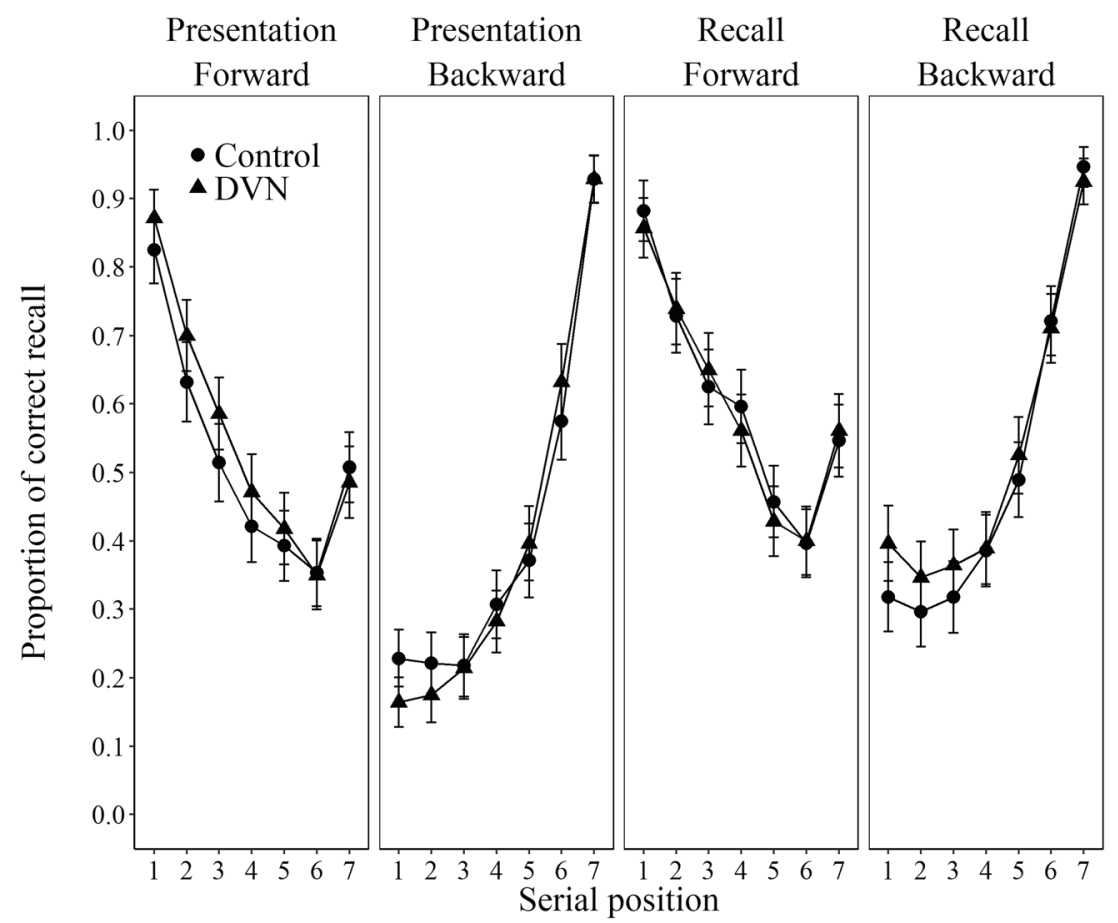

Fig. 4 Proportion of correct recall for Experiment 3 as a function of recall direction (forward vs. backward), locus of the interference (presentation vs. recall), interference task (none vs. dynamic visual noise), and serial position (1-7). Error bars represent 95\% within-participant confidence intervals computed according to Morey's (2008) procedure 
normally relies on visuospatial representations, participants may have switched to verbal representations in the presence of manual tapping; such shifts in strategy could have masked detrimental effects of tapping in backward recall. To address this issue, we added an articulatory suppression requirement in all conditions. In other words, manual spatial tapping was performed with concurrent articulatory suppression and was compared to a control condition in which participants only performed articulatory suppression. Other than the addition of articulatory suppression, the design was the same as in Experiment 2.

\section{Method}

Participants Forty undergraduate students (27 women and 13 men) from Université de Moncton volunteered to participate in exchange for course credits. All reported normal or corrected-to-normal vision and were French speakers. None had participated in the previous experiments.

Materials and procedure The method was the same as in Experiment 2 except that articulatory suppression was required in addition to spatial manual tapping, and recall was manual. More specifically, in all conditions, participants were required to repeat aloud the alphabetic sequence (a, b, c, d, e, f, g) at a pace of two letters per second. For the group experiencing spatial manual tapping at encoding, participants began repeating the letters as soon as the instruction "Parler" (to speak) appeared on the screen and continued until all to-beremembered digits reappeared on the screen for recall. Instructions were on the center of the screen for $1,500 \mathrm{~ms}$, and the presentation of the first digit began immediately after. For tapping trials, the instruction was "Parler + Frappe" (to speak + tapping). Manual spatial tapping was performed with the non-dominant hand at the same time as articulatory suppression. The method was the same when interference occurred at recall, except that participants executed the interfering task during the recall period. Accordingly, at recall, the instructions reappeared simultaneously with the digits to indicate the beginning of the interference task. Participants were warned to continue tapping until the last to-be remembered item has been recalled. Recall in both conditions was achieved by clicking on the items with the dominant hand.

\section{Results}

As can be seen in Figs. 1 and 5 and as confirmed by the ANOVA in Appendix Table 1, recall performance was better in the forward $(M=.58 S D=.32)$ than in the backward recall $(M=.43 S D=.36)$ conditions. Overall, recall performance was significantly lower in the interference (articulatory suppression + spatial manual tapping $)(M=.45 S D=.33)$ than in the control (articulatory suppression) condition $(M=.56 S D=$ .36). Furthermore, the addition of spatial manual tapping had a larger detrimental effect at encoding $(M=.14 S D=.13)$ than at recall $(M=.07 S D=.13)$. The interaction between the locus of the interference and recall direction was significant. This interaction is simply to do with the better recall in the forward than in the backward condition for the recall interference group; recall was similar in both recall directions for the encoding interference group. There was also a significant interaction between the locus of the interference and tapping. This interaction is due to a larger effect of tapping when it occurred at encoding than when it occurred at recall. The interaction between recall direction, interference, and serial position also reached a conventional level of significance. As previously observed in backward recall studies, the latter interaction simply reflects the larger interference effect on early serial positions in forward recall and on later serial positions in backward recall (for a review, see Ritchie et al., 2015). As in Experiment 1, there was a significant interaction between serial position, recall direction, and the locus of the interference. Most importantly, there was no significant interaction between recall direction and interference or between recall direction, interference, and the locus of the interference.

Bayesian paired-samples $\boldsymbol{t}$-tests The null effect of tapping between recall directions for each interference loci was explored with Bayesian paired-samples $t$-tests. Results revealed anecdotal evidence in favor of the null hypothesis (backward interference effect is not greater than forward interference effect), $\mathrm{BF}_{01}=1.35$, when interference occurred during presentation and positive evidence, $\mathrm{BF}_{01}=8.90$, when interference occurred during recall. Importantly, an inspection of the evidence in favor of the alternative hypothesis (backward interference effect is greater than forward interference effect) was inferior to the evidence in favor of the null hypothesis and anecdotal, $\mathrm{BF}_{10}=0.74\left(1 / \mathrm{BF}_{01}\right)$.

\section{Discussion}

As in Experiments 1 and 2, recall accuracy in forward and backward recall was lower in the spatial interference than control condition. Consistent with Experiments 1 and 2, the effect of spatial manual tapping was equally disruptive in backward and in forward recall. Because participants were required to perform articulatory suppression throughout the task, the similar effect of manual tapping in forward and backward recall cannot be attributed to a shift in strategies from a typical visualspatial processing to a verbal processing in backward recall.

\section{Experiment 5}

Results of the current experiments are clear and straightforward: The same pattern of results was always observed in forward and backward recall. These results contradict those 


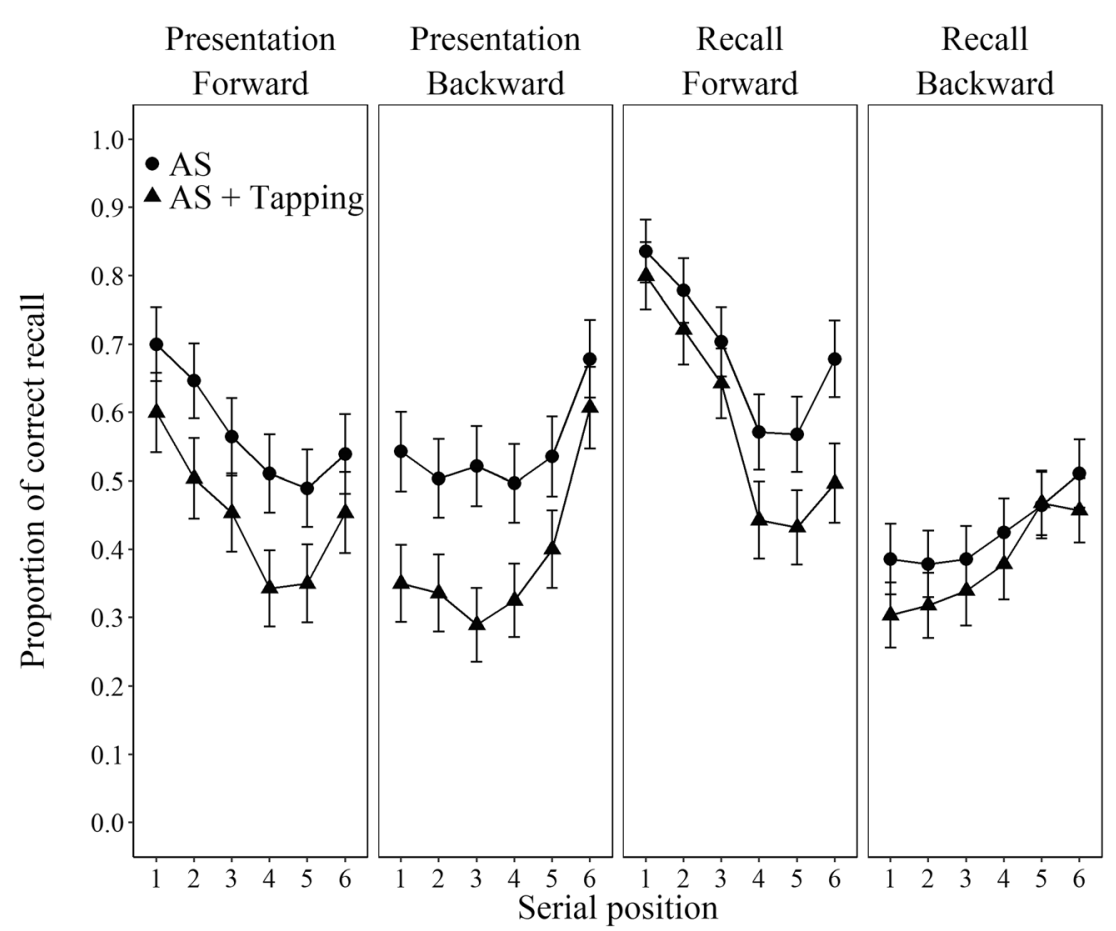

Fig. 5 Proportion of correct recall for Experiment 4 as a function of recall direction (forward vs. backward), locus of the interference (presentation vs. recall), interference task (articulatory suppression vs. articulatory

of St Clair-Thompson and Allen (2013) and Li and Lewandowsky (1995), as well as the given view in the field of intelligence testing (Reynolds, 1997). According to this view, forward and backward recall call upon qualitatively distinct retrieval processes. More precisely, backward recall would rely more heavily on visuospatial processes compared to forward recall. In order to account for these discrepancies, it could be argued that foreknowledge of recall direction may allow participants to encode items differently, as suggested by studies showing that participants use alternative strategies to meet task demands (see, e.g., Miles, Jones, \& Madden, 1991; Watkins, LeCompte, \& Kim, 2000). Under this view, reminiscent of the transfer appropriate processing hypothesis (see Morris, Bransford, \& Franks, 1977), backward recall would only rely on visuospatial processes when enough visual features have been encoded. This would be the case in psychometric tests, as well as in the study by St Clair-Thompson and Allen (2013), in which recall direction was blocked and known in advance. In Experiment 5, we tested this idea by telling participants at the beginning of each block what the direction of recall was going to be for said block. A modified version of the visuospatial hypothesis, taking foreknowledge of recall direction into account, would suggest that manual tapping is more disruptive in backward than in forward recall when recall direction is known. This would occur because, foreknowledge leads to more visuospatial encoding in backward recall relative to forward recall; it follows that tapping should interfere more with backward than with forward recall suppression and tapping), and serial position (1-6). Error bars represent 95\% within-participant confidence intervals computed according to Morey's (2008) procedure

(see, e.g., Guitard \& Saint-Aubin, 2015; Meiser \& Klauer, 1999; Poirier et al., 2019). To test this modified version of the visuospatial hypothesis we used the same design as in Experiment 2, but recall direction was blocked and was specified at the beginning of each block.

\section{Method}

Participants Forty undergraduate students (31 women and nine men) from Université de Moncton volunteered to participate in exchange for course credits. All reported normal or corrected-to-normal vision and were French speakers. None took part to the previous experiments.

Materials and procedure The method was the same as in Experiment 2, except that recall direction varied across blocks. More specifically, recall direction was presented at the beginning of each of the two blocks (forward, backward) and never varied within a block. This procedure is analogous with intelligence tests in which participants separately complete forward and backward digit spans. The order of the blocks was counterbalanced across participants. In addition, items were not represented at recall.

\section{Results}

As can be seen in Figs. 1 and 6 and as confirmed by the ANOVA in Appendix Table 1, recall performance was better 
in the forward $(M=.66 S D=.32)$ than in the backward recall $(M=.56 S D=.29)$ conditions. Overall, recall performance was lower in the spatial manual tapping $(M=.66 S D=.30)$ than in the control condition $(M=.56 S D=.31)$. Furthermore, the addition of spatial manual tapping had a larger detrimental effect at encoding $(M=.14 S D=.09)$ than at recall $(M=.05$ $S D=.06)$. As in Experiment 4, the interaction between the locus of the interference and tapping was significant. This interaction is due to a larger effect of tapping when it occurred at encoding than when it occurred at recall. Most importantly, there was a significant interaction between recall direction, interference, and the locus of the interference. This interaction is due to the similar detrimental effect of tapping in forward and backward recall directions when it was performed at encoding, but to a restricted detrimental effect in the backward recall direction when it was performed at recall.

Bayesian paired-samples $t$-tests The effect of tapping between recall directions for each interference loci was explored with Bayesian paired-samples $t$-tests. Results revealed positive evidence in favor of the null hypothesis (backward interference effect is not greater than forward interference effect), $\mathrm{BF}_{01}=$ 6.81 , when interference occurred during presentation and positive evidence, $\mathrm{BF}_{10}=7.18$, (backward interference effect is greater than forward interference effect) when interference occurred during recall.

\section{Discussion}

Forward and backward recall were equally disrupted by manual tapping when it occurred at encoding. This finding nicely replicated results of the previous experiments reported here. Once more, current results contradict those of St ClairThompson and Allen (2013), who did not observe a disruptive effect on backward recall when interference occurred at encoding. However, when tapping occurred at recall, its selective detrimental effect on backward recall nicely reconciled results of the first four experiments without foreknowledge with those of St Clair-Thompson and Allen and with the visuospatial hypothesis.

\section{General discussion}

The current study was aimed at investigating the visuospatial hypothesis as applied to backward serial recall. According to this hypothesis, forward and backward recall call upon qualitatively distinct retrieval processes (Li \& Lewandowsky, 1993, 1995; Reynolds, 1997; St Clair-Thompson \& Allen, 2013). More specifically, backward recall is thought to rely more heavily on visuospatial processes compared to forward recall. Consequently, a secondary task known to interfere with visuospatial representations should hinder backward recall more than forward recall. In five experiments, we tested this hypothesis by manipulating recall direction (forward vs. backward), locus of interference (encoding vs. recall), the interference task itself (spatial manual tapping vs. DVN), the to-beremembered items (words vs. digits), and the foreknowledge of recall direction (post-cued vs. pre-cued).

Our results are clear and straightforward: Contrary to a strict interpretation of the visuospatial hypothesis, when recall direction is post-cued, DVN had no effect on performance and spatial manual tapping had the same detrimental effect on forward and backward recall. The latter pattern of results was observed with words (Experiment 1) and digits (Experiments 2 and 4); with oral (Experiments 1 and 2) and manual responses (Experiment 4); when interference was introduced at presentation (Experiments 1, 2, 4, and 5) or at recall (Experiments 1, 2, and 4), and even when usage of an alternative phonological strategy was prevented (Experiment 4). However, consistent with the predictions derived from the visuospatial hypothesis, in Experiment 5, when recall direction was known prior to encoding the items, spatial manual tapping performed at recall had a larger detrimental effect on backward recall than forward recall. Taken together, current results provided partial support to the visuospatial hypothesis, while highlighting the need for an additional assumption.

The large and reliable effect of spatial manual tapping on forward recall performance for verbal items replicated previous results (Allen, Havelka, Falcon, Evans, \& Darling, 2015; Guérard \& Tremblay, 2008; Guérard, Jalbert, Neath, Surprenant, \& Bireta, 2009; Guitard \& Saint-Aubin, 2015; Jones et al., 1995, Kee, Bathurst, \& Hellige, 1983; Keefe, 1985; Larsen \& Baddeley, 2003; Meiser \& Klauer, 1999; Poirier et al., 2019; Thornton \& Peters, 1982). In addition, when recall direction was post-cued, the similar detrimental effect of manual tapping in forward and backward recall extends previous results observed with a spatial memory task. Using the Corsi blocks as the primary memory task, Higo, Minamoto, Ikeda, and Osaka (2014) reported an equivalent detrimental effect of spatial manual tapping in both forward and backward recall (see also Vandierendonck, Kemps, Fastame, \& Szmalec, 2004). Spatial manual tapping adds to the list of factors that have been found to have a similar effect in forward and backward recall for post-cued demonstrations, such as phonological similarity, word length, irrelevant speech, lexicality, associative links, and imageability (Beaudry et al., 2018; Guérard \& Saint-Aubin, 2012; Guérard et al., 2012; Saint-Aubin et al., 2014). Finally, the detrimental effect of spatial manual tapping was of similar magnitude when it was performed at encoding or at recall in two experiments (Experiments 1 and 2), and it was larger when performed at encoding than at recall in two experiments (Experiments 4 and 5). This pattern of results is in line with previous forward recall studies. For instance, Meiser and Klauer (1999) observed a detrimental effect at encoding, but 


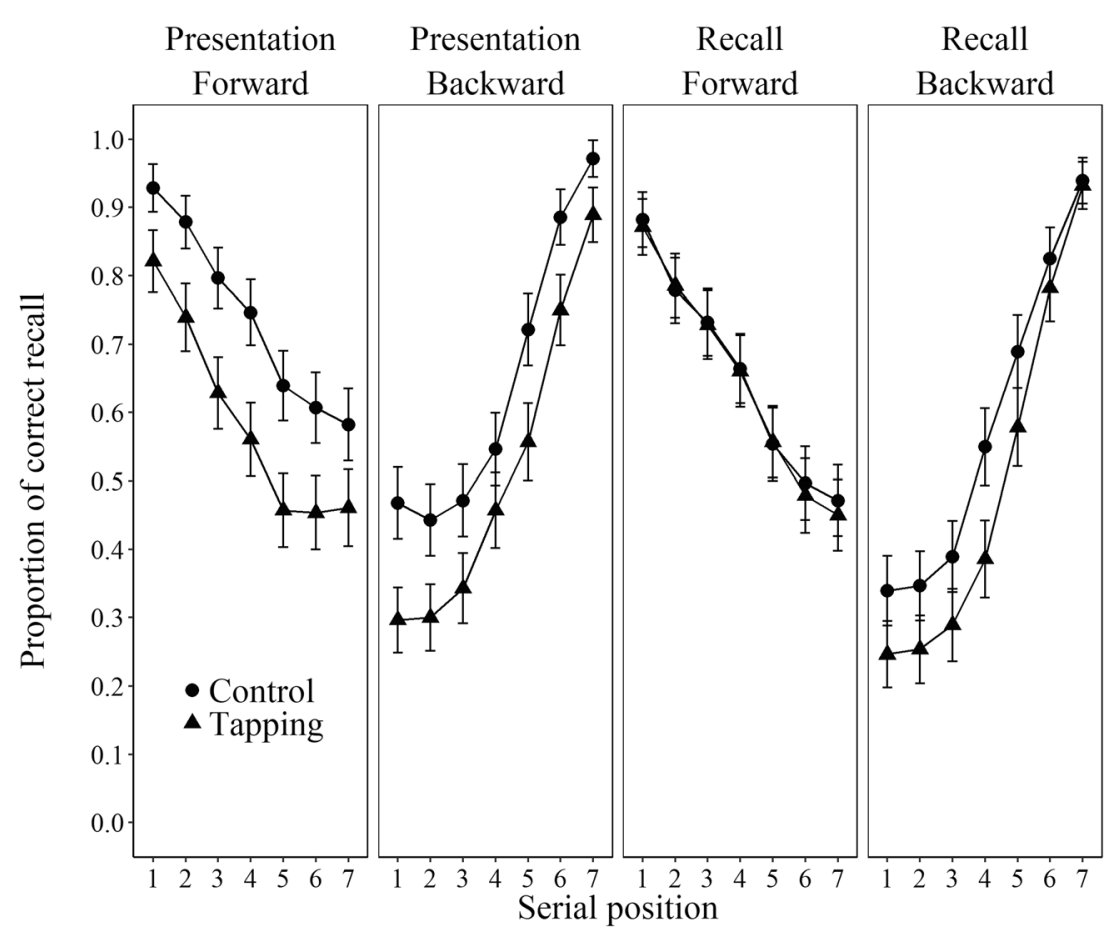

Fig. 6 Proportion of correct recall for Experiment 5 as a function of recall direction (forward vs. backward), locus of the interference (presentation vs. recall), interference task (none vs. spatial manual tapping), and serial

not during a retention interval. However, Allen et al. (2015) found a detrimental effect at both recall and encoding.

In Experiment 3, the failure to find a DVN effect on memory performance for verbal materials adds to the list of studies that failed to find the effect (see, e.g., Avons \& Sestieri, 2005; Andrade, Kemps, Werniers, May, \& Szmalec, 2002; Chubala et al., 2018; Quinn \& McConnell, 2006; St Clair-Thompson and Allen, 2013). Our results fit well with those of Quinn and McConnell (1996), who showed that DVN interferes with the encoding, maintenance, and retrieval of verbal materials if, and only if, participants engaged in elaborated visual mnemonic processing such as paring each to-remembered-item with an image. In line with those results, in their fourth experiment, St Clair-Thompson and Allen only observed a DVN effect among a subgroup of 11 participants who reported having used visual strategies. It is worth noting that in itself, this result is contrary to the visuo-processing hypothesis as twothirds of the participants were classified as not having used those strategies in backward recall. Although interesting, results with the subgroup of 11 participants can be interpreted by calling upon a reverse causality. Overall, DVN would have no effect. As any randomly distributed variable, some participants would perform worse in the DVN than the control condition, while it would be the reverse for others. When questioned about their strategies, participants who struggled more with the DVN in backward recall may have mentioned using a visual strategy as it was allowing them to make sense of their performance. position (1-7). Error bars represent 95\% within-participant confidence intervals computed according to Morey's (2008) procedure

In their fifth experiment, St Clair-Thompson and Allen (2013) found a significant detrimental effect of DVN in backward recall with the whole sample and no effect in forward recall. However, since performance was at ceiling in the forward recall condition, it is unclear if the observed DVN effect was due to the involvement of visual processes in backward recall or if it simply created some general distraction, which could not be observed in forward recall due to the ceiling effect. When considering all evidence from the current and past studies, it can be concluded that, unless participants are forced to use a visual strategy with verbal materials, the effect of DVN on immediate serial recall is at best small and elusive.

The current results fit well with some of those reported by $\mathrm{Li}$ and Lewandowsky (1995). In their first two experiments, after the presentation of each list item, participants performed one trial of a mental rotation task adapted from Shepard and Metzler (1971). Results showed a large detrimental effect in both forward and backward recall and the magnitude of the effect was the same for both recall directions. This result is interesting because the mental rotation task is well known to call upon visuospatial processing (see, e.g., Gignac, Kovacs, \& Reynolds, 2018). Although Li and Lewandowsky offered an alternative interpretation, this finding can be seen as contradicting the visuospatial hypothesis. Furthermore, these results are consistent with the similar detrimental effect of spatial manual tapping when introduced at encoding for both preand post-cued manipulations. 
The main support for the visuospatial hypothesis was provided by the last three experiments of Li and Lewandowsky (1995). In two experiments, they found that presenting the to-be-remembered items at various locations on the screen instead of presenting them in the center of the screen interfered with backward but not forward recall. The lack of effect in forward recall is surprising in light of results showing binding between verbal and spatial representations in forward recall. For instance, Guérard et al. (2009) have shown a detrimental effect of phonological similarity among the to-be-remembered letters presented at various locations on the screen when participants were asked to recall the locations instead of the letters. Furthermore, although fitting well with the visuospatial hypothesis, results of $\mathrm{Li}$ and Lewandowsky are hard to reconcile with subsequent findings. For instance, Fischer-Baum and Benjamin (2014) used an immediate serial recall task in which participants performed forward or backward recall. The to-be-remembered letters were sequentially presented in a unique spatial position, with the order of these spatial positions progressing from either left to right or right to left. In forward and backward recall conditions, recall was superior when the sequence of letters was presented from left to right (congruent with English reading) compared to presentation from the right to the left (incongruent with English reading). Most importantly, in line with current results, and contrary to the $\mathrm{Li}$ and Lewandowsky results, the magnitude of the effect was similar for both recall directions. In their last experiment, Li and Lewandowsky reported a beneficial effect of visual similarity in backward, but not in forward recall. This finding diverges from later studies, which all showed a detrimental effect of visual similarity on forward recall of verbal materials (see, e.g., Logie et al., 2000; Logie et al., 2016; Poirier et al., 2007; Saito et al., 2008). The reason for these discrepancies is not fully understood, but they weakened the support for the visuospatial processing hypothesis.

\section{Theoretical implications}

Our study was designed to test the visuospatial hypothesis. According to this hypothesis, backward recall relies in part on visuospatial processes. For instance, participants would create a mental image of the list, and at recall, would either scan it to produce the items or execute a variant of a mental rotation to produce the items in their requested order (Hermelin \& O'Connor, 1973; Li \& Lewandowsky, 1993, 1995; O'Connor \& Hermelin, 1973; St Clair-Thompson \& Allen, 2013). According to the visuospatial hypothesis, manual tapping should have interfered more with backward than forward recall independently of the foreknowledge of the recall direction. Furthermore, according to St Clair-Thompson \& Allen, the larger detrimental effect in backward recall should only emerge when the interference task is introduced at recall. Consistent with this hypothesis, whether recall direction was pre- or post-cued, when manual tapping was performed at encoding, we observed a similar detrimental effect in forward and backward recall conditions. Critically, in Experiment 5, when recall direction was post-cued and manual tapping was performed at recall, a larger detrimental effect was observed in backward than in forward recall. However, contrary to the predictions of the visuospatial hypothesis, when recall direction was post-cued, the detrimental effect of spatial manual tapping was of similar magnitude in forward and backward recall (see also Fischer-Baum \& Benjamin, 2014).

These results highlight the need to qualify the visuospatial hypothesis. Based on the transfer-appropriate processing view, it could be argued that backward recall relies more on visuospatial processes, if and only if, enough relevant features were encoded (see Morris et al., 1977, for a similar view). As previously suggested, even for verbal materials, there may be both phonological and visuospatial encoding (see, e.g., Doherty et al., 2018). When anticipating forward recall, participants would rely more on phonological encoding and they would rely more on visuospatial encoding when anticipating backward recall (for a related idea see, Miles et al. 1991; Watkins et al., 2000). When unsure about recall direction, participants would rely on an intermediate level of phonological and visuospatial encoding. This would translate into a similar detrimental effect of manual tapping - or any other visuospatial interfering task - if participants have no foreknowledge of recall direction and a much larger effect when they anticipate a backward than a forward recall (see Logie, 2018).

\section{Conclusion}

The current study uncovers a complex but coherent interaction between foreknowledge, recall direction, visuospatial interference, and its locus. These findings do not support a strict version of the visuospatial hypothesis. However, a modified version in which retrieval processes are dependent upon encoding provides an adequate account. Although more evidence is needed to evaluate this modified version of the visuospatial hypothesis, the latter adequately accounts for all available findings.

Authors' Note This research was supported by a Natural Sciences and Engineering Research Council of Canada graduate scholarship to Dominic Guitard and by a Discovery grant from the Natural Sciences and Engineering Research Council of Canada to Jean Saint-Aubin.

Open Practices Statement All the data and materials for the experiments reported here are available upon request by contacting the corresponding author. None of the experiments was preregistered. 


\section{Appendix}

\section{Results of Experiments 1-5}

Table 1 Analyses of variance for the proportion of correct recall (strict recall criterion)

\begin{tabular}{|c|c|c|c|c|c|c|c|c|c|c|c|c|}
\hline \multirow[t]{2}{*}{ Source } & \multicolumn{4}{|c|}{ Tapping (Exp.1 Post-cued) } & \multicolumn{4}{|c|}{ Tapping (Exp.2 Post-cued) } & \multicolumn{4}{|c|}{ DVN (Exp.3 Post-cued) } \\
\hline & $d f$ & $F$ & $P$ & $\eta_{p}^{2}$ & $d f$ & $F$ & $p$ & $\eta_{p}^{2}$ & $d f$ & $F$ & $p$ & $\eta_{p}^{2}$ \\
\hline LI & 1,38 & 0.36 & .55 & .01 & 1,38 & 2.45 & .13 & .06 & 1,38 & 2.22 & .15 & .06 \\
\hline $\mathrm{RD}$ & 1,38 & 0.05 & .83 & .00 & 1,38 & 34.90 & $<.001$ & .48 & 1,38 & 34.90 & $<.001$ & .48 \\
\hline I & 1,38 & 15.04 & $<.001$ & .28 & 1,38 & 54.17 & $<.001$ & .59 & 1,38 & 1.82 & .19 & .05 \\
\hline SP & $* 2.53,96.24$ & 30.86 & $<.001$ & .45 & $* 2.80,106.48$ & 19.92 & $<.001$ & .34 & $* 2.51,95.30$ & 51.35 & $<.001$ & .56 \\
\hline LI x RD & 1,38 & 0.71 & .40 & .02 & 1,38 & 0.26 & .62 & .01 & 1,38 & 1.20 & .28 & .03 \\
\hline LI x I & 1,38 & 2.10 & .16 & .05 & 1,38 & 0.45 & .51 & .01 & 1,38 & 0.02 & .90 & .00 \\
\hline LI x SP & $* 2.53,96.24$ & 0.26 & .82 & .01 & $* 2.80,106.48$ & 1.94 & .13 & .05 & $* 2.51,95.30$ & 0.77 & .49 & .02 \\
\hline $\mathrm{RD} \times \mathrm{I}$ & 1,38 & 1.27 & .27 & .03 & 1,38 & 0.17 & .68 & .01 & 1,38 & 0.07 & .79 & .00 \\
\hline $\mathrm{RD} \times \mathrm{SP}$ & $* 4.22,160.21$ & 98.28 & $<.001$ & .72 & $* 3.02,114.59$ & 90.62 & $<.001$ & .71 & $* 1.87,70.90$ & 162.81 & $<.001$ & .81 \\
\hline I x SP & 6,228 & 1.59 & .17 & .04 & 6,228 & 0.16 & .98 & .01 & $* 4.40,167.30$ & 0.90 & .47 & .02 \\
\hline LI x RD x I & 1,38 & 0.79 & .38 & .02 & 1,38 & 0.02 & .90 & .00 & 1,38 & 3.41 & .07 & .08 \\
\hline LI x RD x SP & $* 4.22,160.21$ & 2.91 & .02 & .07 & $* 3.02,114.59$ & 0.63 & .60 & .02 & $* 1.87,70.90$ & 1.35 & .26 & .03 \\
\hline LI x I x SP & 6,228 & 1.04 & .40 & .03 & 6,228 & 0.85 & .52 & .02 & $* 4.40,167.30$ & 0.75 & .57 & .02 \\
\hline RD x I x SP & 6,228 & 0.41 & .85 & .01 & 6,228 & 1.13 & .35 & .03 & 6,228 & 0.67 & .63 & .02 \\
\hline LI x RD x I x SP & 6,228 & 0.61 & .71 & .02 & 6,228 & 1.49 & .20 & .04 & 6,228 & 2.90 & .02 & .07 \\
\hline \multirow[t]{2}{*}{ Source } & \multicolumn{4}{|c|}{ AS + Tapping (Exp.4 Post-cued) } & \multicolumn{4}{|c|}{ Tapping (Exp.5 Pre-cued) } & & & & \\
\hline & $d f$ & $F$ & $P$ & $\eta_{p}^{2}$ & $d f$ & $F$ & $p$ & $\eta_{p}^{2}$ & & & & \\
\hline LI & 1,38 & 0.32 & .58 & .01 & 1,38 & 0.25 & .62 & .01 & & & & \\
\hline $\mathrm{RD}$ & 1,38 & 23.39 & $<.001$ & .38 & 1,38 & 15.54 & $<.001$ & .29 & & & & \\
\hline I & 1,38 & 47.56 & $<.001$ & .56 & 1,38 & 57.39 & $<.001$ & .60 & & & & \\
\hline SP & $* 2.55,96.88$ & 13.94 & $<.001$ & .27 & $* 3.45,131.09$ & 29.06 & $<.001$ & .43 & & & & \\
\hline LI x RD & 1,38 & 10.45 & .003 & .22 & 1,38 & 0.26 & .61 & .01 & & & & \\
\hline LI x I & 1,38 & 4.86 & .03 & .11 & 1,38 & 14.39 & $<.001$ & .28 & & & & \\
\hline LI x SP & $* 2.55,96.88$ & 1.52 & .22 & .04 & $* 3.45,131.09$ & 0.62 & .62 & .02 & & & & \\
\hline $\mathrm{RD} \times \mathrm{I}$ & 1,38 & 0.09 & .77 & .00 & 1,38 & 2.04 & .16 & .06 & & & & \\
\hline $\mathrm{RD} \times \mathrm{SP}$ & $* 3.41,129.58$ & 48.34 & $<.001$ & .56 & *1.93,73.26 & 149.87 & $<.001$ & .80 & & & & \\
\hline $\mathrm{I} \times \mathrm{SP}$ & 5,190 & 0.58 & .69 & .02 & $* 4.12,156.51$ & 1.45 & .22 & .04 & & & & \\
\hline LI x RD x I & 1,38 & 3.09 & .09 & .08 & 1,38 & 5.90 & .02 & 13 & & & & \\
\hline LI x RD x SP & $* 3.41,129.58$ & 3.64 & .01 & .09 & $* 1.93,73.26$ & .87 & .42 & .02 & & & & \\
\hline LI x I x SP & 5,190 & 3.69 & .01 & .09 & $* 4.12,156.51$ & .51 & .73 & .01 & & & & \\
\hline RD x I x SP & $* 3.71,141.15$ & 2.85 & .03 & .07 & $* 3.59,136.39$ & 1.19 & .32 & .03 & & & & \\
\hline LI x RD x I x SP & $* 3.71,141.15$ & 0.42 & .78 & .01 & $* 3.59,136.39$ & 1.84 & .13 & .05 & & & & \\
\hline
\end{tabular}

Note. Exp. = experiment; DVN = dynamic visual noise; $\mathrm{AS}=$ articulatory suppression; $\mathrm{LI}=$ locus of the interference task; $\mathrm{RD}=$ recall direction; $\mathrm{I}=$ interference task; SP = serial position; Post-Cued = recall direction was known after the presentation of the test items; Pre-Cued = recall direction was known before the presentation of the test items

Results in bold indicate that $p<.05$

*Mauchly's test of sphericity indicates that the assumption of sphericity is violated $p<.05$ and the Grenhouse-Geisser correction is applicated 


\section{References}

Allen, R. J., Havelka, J., Falcon, T., Evans, S., \& Darling, S. (2015). Modality specificity and integration in working memory: Insights from visuospatial bootstrapping. Journal of Experimental Psychology: Learning, Memory, and Cognition, 41, 820-830.

Andrade, J., Kemps, E., Werniers, Y., May, J., \& Szmalec, A. (2002). Insensitivity of visual short term memory to irrelevant visual information. The Quarterly Journal of Experimental Psychology A: Human Experimental Psychology, 55A, 753-774.

Avons, S. E., \& Sestieri, C. (2005). Dynamic visual noise: No interference with visual short-term memory or the construction of visual images. European Journal of Cognitive Psychology, 17, 405-424.

Baker, R., Tehan, G., \& Tehan, H. (2012). Word length and age influences on forward and backward immediate serial recall. Memory \& Cognition, 40, 40-51.

Beaudry, O., Saint-Aubin, J., Guérard, K., \& Pâquet, M. (2018). Are lexical factors immune to response modality in backward recall? The effects of imageability and word frequency. Canadian Journal of Experimental Psychology.

Bireta, T. J., Fry, S. E., Jalbert, A., Neath, I., Surprenant, A. M., Tehan, G., \& Tolan, G. A. (2010). Backward recall and benchmark effects of working memory. Memory \& Cognition, 38, 279-291.

Blankenship, A.B. (1938). Memory span: A review of the literature. Psychological Bulletin, 35, 1-25.

Bopp, K. L., \& Verhaeghen, P. (2005). Aging and verbal memory span: A meta-analysis. The Journals of Gerontology Series B: Psychological Sciences and Social Sciences, 60(5), P223-P233.

Bull, R., Espy, K. A., \& Wiebe, S. A. (2008). Short-term memory, working memory, and executive functioning in preschoolers: Longitudinal predictors of mathematical achievement at age 7 years. Developmental Neuropsychology, 33, 205-228.

Chatham, C. H., Herd, S. A., Brant, A. M., Hazy, T. E., Miyake, A., O Reilly, R., \& Friedman, N. P. (2011). From an executive network to executive control: A computational model of the n-back task. Journal of Cognitive Neuroscience, 23, 3598-3619.

Chubala, C., Surprenant, A. M.. Neath, I., \& Quinlan, P. T. (2018). Does dynamic visual noise eliminate the concreteness effect in working memory? Journal of Memory and Language, 102, 97-114. http://dx. doi.org/10.1016/j.jml.2018.05.009

Colom, R., Abad, F. J., Rebello, I., \& Shih, P. C. (2005). Memory span and general intelligence: A latent variable approach. Intelligence, $33,623-624$

Dehaene, S., Bossini, S., \& Giraux, P. (1993). The mental representation of parity and number magnitude. Journal of Experimental Psychology: General, 122, 371-396.

Doherty, J. M., Belletier, C., Rhodes, S., Jaroslawska, A., Barrouillet, P., Camos, V., ... Logie, R. H. (2018). Dual-task costs in working memory: An adversarial collaboration. Journal of Experimental Psychology: Learning, Memory, and Cognition.

Donolato E., Giofrè D., \& Mammarella I. C. (2017) Differences in Verbal and Visuospatial Forward and Backward Order Recall: A Review of the Literature. Front. Psychol. 8:663.

Elliot, C. D. (1996). British Ability Scales II. Windsor: NFER-Nelson

Fischer-Baum, S., \& Benjamin, A. S. (2014). Time, space, and memory for order. Psychonomic Bulletin \& Review, 21, 1263-1271.

Folstein, M., Folstein, S.E., McHugh, P.R. (1975). "Mini-Mental State" a practical method for grading the cognitive state of patients for the clinician. Journal of Psychiatric Research, 12, 189-198.

Gathercole, S. E., Pickering, S. J., Knight, C., \& Stegmann, Z. (2004). Working memory skills and educational attainment: Evidence from national curriculum assessments at 7 and 14 years of age. Applied Cognitive Psychology, 18(1), 1-16.

Gerton, B. K., Brown, T. T., Meyer-Lindenberg, A., Kohn, P., Holt, J. L., Olsen, R. K., \& Berman, K. F. (2004). Shared and distinct neurophysiological components of the digits forward and backward tasks as revealed by functional neuroimaging. Neuropsychologia, 42, 1781-1787.
Gignac, G. E., Kovacs, K., \& Reynolds, M. R. (2018). Backward and forward serial recall across modalities: An individual differences perspective. Personality and Individual Differences, 121, 147-151.

Guérard, K., Jalbert, A., Neath, I., Surprenant, A. M., \& Bireta, T. J. (2009). Irrelevant tapping and the acoustic confusion effect: The effect of spatial complexity. Experimental Psychology, 56, 367-374.

Guérard, K., \& Tremblay, S. (2008). Revisiting evidence for modularity and functional equivalence across verbal and spatial domains in memory. Journal of Experimental Psychology-Learning Memory and Cognition, 34, 556-569.

Guérard, K., Tremblay, S., \& Saint-Aubin, J. (2009). Similarity and binding in memory: Bound to be detrimental. The Quarterly Journal of Experimental Psychology, 62, 26-32.

Guérard, K. \& Saint-Aubin, J. (2012). Assessing the effect of lexical variables in backward recall. Journal of Experimental Psychology: Learning, Memory, \& Cognition, 38, 142-152.

Guérard, K., Saint-Aubin, J., Burns, S. C., \& Chamberland, C. (2012). Revisiting backward recall and benchmark memory effects: A reply to Bireta et al. (2010). Memory \& Cognition, 40, 388-407.

Guitard, D., \& Saint-Aubin, J. (2015). A Replication of "Functional equivalence of verbal and spatial information in serial short-term memory (1995; Experiments 2 and 3)". The Quantitative Methods for Psychology, 11, 4-7.

Hanley, J. R., Young, A. W., \& Pearson, N. A. (1991). Impairment of the visuo-spatial sketch pad. The Quarterly Journal of Experimental Psychology A: Human Experimental Psychology, 43A, 101-125.

Henson, R. N. A. (1998). Short-term memory for serial order: The StartEnd Model. Cognitive Psychology, 36, 73-137.

Hermelin, B., \& O'Connor, N. (1973). Ordering in recognition memory after ambiguous initial or recognition displays. Canadian Journal of Psychology, 27, 191.

Higo K, Minamoto T, Ikeda T and Osaka M (2014) Robust order representation is required for backward recall in the Corsi blocks task. Frontiers in Psychology 5:1285. https://doi.org/10.3389/fpsyg. 2014.01285

Hoshi, Y, Oda, I, Wada, Y, Ito, Y, Yamashita, Y, Oda, M, Ohta, K, Yamada, Y, \& Tamura, M. (2000). Visuospatial imagery is a fruitful strategy for the digit span backward task: a study with near infrared optical tomography. Cognitive Brain Research, 9, 339-342.

Hulme, C., Roodenrys, S., Schweickert, R., Brown, G. D. A., Martin, S., \& Stuart, G. (1997). Word-frequency effects on short-term memory tasks: Evidence for a redintegration process in immediate serial recall. Journal of Experimental Psychology: Learning, Memory, and Cognition, 23, 1217-1232.

Jeffreys, H. (1961). Theory of probability ( $3^{\text {rd }}$ edition). New York: Oxford University Press.

Jones, D., Farrand, P., Stuart, G., \& Morris, N. (1995). Journal of Experimental Psychology: Learning, Memory, and Cognition. 21, 1008-1018.

Kass, R., \& Raftery, A. (1995). Bayes Factors. Journal of the American Statistical Association, 90, 773-795. https://doi.org/10.2307/ 2291091

Kee, D. W., Bathurst, K., \& Hellige, J. B. (1983). Lateralized interference of repetitive finger tapping: Influence of familial handedness, cognitive load and verbal production. Neuropsychologia, 21, 617-624.

Keefe, K. (1985). Motor and cognitive interference effects on unimanual tapping rates. Brain and Cognition, 4, 165-170.

Larsen, J. D., \& Baddeley, A. (2003). Disruption of verbal stm by irrelevant speech, articulatory suppression, and manual tapping: Do they have a common source? The Quarterly Journal of Experimental Psychology Section A, 56, 1249-1268.

Lewandowsky, S., \& Farrell, S. (2008). Short-Term Memory : New Data and a Model. Psychology of Learning and Motivation, 49, 1-48.

Li, S.-C., \& Lewandowsky, S. (1993). Intralist distractors and recall direction: Constraints on models of memory for serial order. Journal 
of Experimental Psychology: Learning, Memory, and Cognition, 19, 895-908. https://doi.org/10.1037/0278-7393.19.4.895

Li, S.-C., \& Lewandowsky, S. (1995). Forward and Backward Recall: Different Retrieval Processes. Journal of Experimental Psychology: Learning, Memory, and Cognition, 21, 837-847.

Logie, R. H. (2018). Human cognition: Common principles and individual variation. Journal of Applied Research in Memory and Cognition, 7, 471-486.

Logie, R. H., Del Sala, S., Wynn, V., \& Baddeley, A. D. (2000). Visual Similarity Effects in Immediate Verbal Serial Recall. The Quarterly Journal of Experimental Psychology Section A, 53, 626-646.

Logie, R. H., Saito, S., Morita, A., Varma, S., \& Norris, D. (2016). Recalling visual serial order for verbal sequences. Memory \& Cognition, 44, 590-607.

Muangpaisan, W., Intalapaporn S., \& Assantachai P. (2010). Digit span and verbal fluency tests in patients with mild cognitive impairment and normal subjects in Thai-community. Journal of the Medical Association of Thailand, 93, 224-230.

Meiser, T., \& Klauer, K. C. (1999). Working memory and changing-state hypothesis. Journal of Experimental Psychology: Learning, Memory, and Cognition, 25, 1272-1299.

Miles, C., Jones, D. M., \& Madden, C. A. (1991). Locus of the irrelevant speech effect in short term memory. Journal of Experimental Psychology: Learning, Memory, and Cognition, 17, 578-584.

Morey, R. D. (2008). Confidence intervals from normalized data: A correction to Cousineau (2005). Tutorial in Quantitative Methods for Psychology, 4, 61-64.

Morris, C. D., Bransford, J. D., \& Franks, J. J. (1977). Levels of processing versus transfer appropriate processing. Journal of Verbal Learning and Verbal Behavior, 16, 519 -533.

New, B., Pallier, C., Brysbaert, M., \& Ferrand, L. (2004). Lexique 2: A new French lexical database. Behavior Research Methods, Instruments \& Computers, 36, 516-524.

Oberauer, K., Lewandowsky, S., Awh, E., Brown, G. D. A., Conway, A., Cowan, N.,... Ward, G. (2018). Benchmarks for models of short term and working memory. Psychological Bulletin, 144, 885-958.

O'Connor, N., \& Hermelin, B. M. (1973). The spatial or temporal organization of short-term memory. The Quarterly Journal of Experimental Psychology, 25, 335-343.

Page, M. A., \& Norris, D. (1998). The primacy model: A new model of immediate serial recall. Psychological Review, 105, 761-781.

Pickering, S. J., \& Gathercole, S. E. (2001). Working Memory Test Battery for Children. London: Psychological Corporation

Poirier, M., Yearsley, J. M., Saint-Aubin, J., Fortin, C., Gallant, G., \& Guitard, D. (2019). Dissociating visuo-spatial and verbal working memory: It's all in the features. Memory \& Cognition, 47, 603-618.

Poirier, M., Saint-Aubin, J., Musselwhite, K., Mohanadas, T., \& Mahammed, G. (2007). Visual similarity effects on short-term memory for order: The case of verbally labeled pictorial stimuli. Memory \& Cognition, 35, 711-723.

Quinn, J. G., \& McConnell, J. (1996). Irrelevant pictures in visual working memory. The Quarterly Journal Of Experimental Psychology A: Human Experimental Psychology, 49A, 200-215.

Quinn, J. G., \& McConnell, J. (2006). The interval for interference in conscious visual imagery. Memory, 14, 241-252.

Reynolds, C. R. (1997). Forward and backward memory span should not be combined for clinical analysis. Archives of Clinical Neuropsychology, 12, 29-40.

Ritchie, G., Tolan, G. A., Tehan, G., Goh, H. E., Guérard, K., \& SaintAubin, J. (2015). Phonological effects in forward and backward serial recall: Qualitative and quantitative differences. Canadian Journal of Experimental Psychology, 69, 95-103.

Rosen, V. M., \& Engle, R. W. (1997). The role of working memory capacity in retrieval. Journal of Experimental Psychology: General, 126, 211-227.
Rudel, R. G., \& Denckla, M. B. (1974). Relation of forward and backward digit repetition to neurological impairment in children with learning disabilities. Neuropsychologia, 12, 109-118.

Saint-Aubin, J., Guérard, K., Chamberland, C., \& Malenfant, A. (2014). Delineating the contribution of long-term associations to immediate recall. Memory, 22, 360-373. https://doi.org/10.1080/09658211. 2013.794242

St Clair-Thompson, H. L., \& Allen, R. J. (2013). Are forward and backward recall the same? A dual-task study of digit recall. Memory \& Cognition, 41, 519-532.

Saito, S., Logie, R. H., Morita, A., \& Law, A. (2008). Visual and phonological similarity effects in verbal immediate serial recall: A test with kanji materials. Journal of Memory and Language, 59, 1-17.

Salamé, P., \& Baddeley, A. D. (1986). Phonological factors in STM: Similarity and the unattended speech effect. Bulletin of the Psychonomic Society, 24, 263-265. https://doi.org/10.3758/ BF03330135

Shepard, R. N., \& Metzler, J. (1971). Mental rotation of threedimensional objects. Science, 171, 701-703.

Surprenant, A. M., Brown, M. A., Jalbert, A., Neath, I., Bireta, T. J., \& Tehan, G. (2011). Backward recall and the word length effect. The American Journal of Psychology, 124, 75-86. http://dx.doi.org/10. 5406/amerjpsyc.124.1.0075

Tehan, G., \& Mills, K. (2007). Working memory and short-term memory storage: What does backward recall tell us? In N. Osaka, R. Logie, \& M. D'Esposito (Eds.), The cognitive neuroscience of working memory (pp. 153-163). Oxford: Oxford University Press.

Thornton, C. D., \& Peters, M. (1982). Interference between concurrent speaking and sequential finger tapping: Both hands show a performance decrement under both visual and non visual guidance. Neuropsychologia, 20, 163-169.

Tremblay, S., Saint-Aubin, J., \& Jalbert, A. (2006). Rehearsal in serial memory for visual-spatial information: Evidence from eye movements. Psychonomic Bulletin \& Review, 13, 452-457. https://doi. org/10.3758/BF03193869

Vallar, G., \& Papagno, C. (1986). Phonological short-term store and the nature of the recency effect: Evidence from neuropsychology. Brain and Cognition, 5, 428-442. http://dx.doi.org/10.1016/02782626(86)90044-8

Vandierendonck, A., Kemps, E., Fastame, M. C., \& Szmalec, A. (2004). Working memory components of the Corsi blocks task. British Journal of Psychology, 95, 57-79.

Wagenmakers, E.-J., Marsman, M., Jamil, T., Ly, A., Verhagen, J., Love, J., .. Morey, R. D. (2018). Bayesian inference for psychology Part I: Theoretical advantages and practical ramifications. Psychonomic Bulletin \& Review, 25, 35-57

Wagenmakers, E.-J., Love, J., Marsman, M., Jamil, T., Ly, A., Verhagen, J., ... Morey, R. D. (2018). Bayesian inference for psychology Part II: Example applications with JASP. Psychonomic Bulletin \& Review, 25, 58-76.

Walker, I., \& Hulme, C. (1999). Concrete words are easier to recall than abstract words: Evidence for a semantic contribution to short-term serial recall. Journal of Experimental Psychology: Learning, Memory, and Cognition, 25, 1256-1271.

Watkins, M. J., LeCompte, D. C., \& Kim, K. (2000). Role of study strategy in recall of mixed lists of common and rare words. Journal of Experimental Psychology: Learning, Memory, and Cognition, 26, 239-245.

Wechsler, D. (2008). Wechsler Adult Intelligence Scale_Fourth Edition. San Antonio, TX: Pearson.

Publisher's note Springer Nature remains neutral with regard to jurisdictional claims in published maps and institutional affiliations. 